\title{
Effects Assessment of Auricular Acupuncture and Group Counseling for Smoking Cessation in Senior High School Students
}

\author{
Pei-Ying Chou, Chen-Jei Tai, You-Jen Tang \\ Taipei Medical University Hospital, Taipei, Taiwan
}

\begin{abstract}
Smoking is a serious public health problem since it harms not only the smokers but also other people in touch with the smokers. Many obstinate illnesses such as cancer, chronic obstructive pulmonary disease, cardiovascular disease are now be proven to be related to smoking itself. To help young smokers, particularly adolescent, quitting smoking can prevent more tobacco use. Some studies showed auricular acupuncture (AA) is increasingly used to treat chemical dependency and withdrawal symptoms based as it's safe and effective. Moreover, group counseling can motivate young adults to set a quit date and strengthen their belief in quitting smoking via peer effects. The present study evaluates the effects of a 8-week smoking cessation courses consisting of AA and group counseling once per week on senior high school students aged from 15 to 18. The AA treatment was administrated at both side of ears on a weekly basis for six weeks. Clinicians gave an intensive counseling session assessing the readiness to quit, exploring smoking habits, and identifying barriers to smoking cessation and relapsing factors. Before each intervention, the 23 participants completed questionnaires and offered levels of carbon monoxide exhalation. The participants showed significant differences in levels of carbon monoxide exhalation, daily tobacco consumptions, reduction in the taste of tobacco and intensity of the desire to smoke after 6-week consecutive sessions. However, the smoking cessation success was only three cases (13\%). Given limited and uncertain efficacy of AA for smoking cessation, though it's non-invasive and safe, it's better to combine AA with other methods not only for synergistic effects but also better smoking cessation success rate.
\end{abstract}

Keywords: tobacco, smoking cessation, auricular acupuncture, adolescent

\section{Introduction}

Nicotiana tabacum brought from North America to Europe by Columbus in 1492 has been responsible for more deaths than any other herb, though it was originally used to treat various conditions based on potential therapeutic properties (Charlton, 2004). Tobacco use, which can be preventable, is strongly related to a cause of non-communicable disease and premature heath, with nearly six to seven million deaths resulted from smoking

\footnotetext{
Pei-Ying Chou, Ph.D. student,, Department of Traditional Chinese Medicine, Taipei Medical University Hospital; Ph.D. Program in Clinical Drug Development of Chinese Herbal Medicine, Taipei Medical University, Taipei, Taiwan.

Chen-Jei Tai, Ph.D. degree, Department of Traditional Chinese Medicine, Taipei Medical University Hospital; Ph.D. Program in Clinical Drug Development of Chinese Herbal Medicine, Taipei Medical University; School of Pharmacy, College of Pharmacy, Taipei Medical University, Taipei, Taiwan.

You-Jen Tang, B.S. degree, Department of Traditional Chinese Medicine, Taipei Medical University Hospital, Taipei, Taiwan.
} 
worldwide each year (D’Angelo, Ahluwalia, Pun, Yin, Palipudi, \& Mbulo, 2016; Pedro, Brito, \& Barros, 2017). Approximately half of all smokers end up with smoking-correlated diseases such as chronic obstructive pulmonary disease (including bronchitis or emphysema), lung cancer, coronary heart disease, or infective lung diseases which may burden society with $\$ 156$ billion in lost productivity, and $\$ 133$ billion in medical service charges in the United States annually (Poulsen, Spillemose, Nielsen, Hergel, Wedell-Wedellsborg, Strand, \& Ringbak, 2015; Vickerman, Zhang, Malarcher, Mowery, \& Nash, 2015).

Almost all tobacco use starts from youth and young adulthood which before age 18 years and is considered as an unsafe action, since the adolescent is a period of great exposure and vulnerability to consumption of tobacco (D’Angelo et al., 2016; Jamal, Gentzke, Hu, Cullen, Apelberg, Homa, \& King, 2017; Andrade, Ferreira, Ramos, Ramos, Scarabottolo, Saraiva, Gobbo, \& Christofaro, 2017). Low education attainment and socioeconomic level, parental smoking (especially mother), tobacco advertisement or promotion, friends and peers smoking, alcohol use could be the contributing factors for tobacco use of the adolescents (Andrade et al., 2017; Haug, Paz Castro, Kowatsch, Filler, \& Schaub, 2017; Xiao, Feng, Jiang, Zhang, \& Liu, 2017). Many studies concluded that smoking adolescents suffer from nicotine dependence may have worse performance on standardized tests, poorer physical activity, alcohol abuse and insomnia when comparing with their non-smoking peers (Sabado, Haynie, Gilman, Simons-Morton, \& Choi, 2017). Moreover, it is estimated that $21.5 \%$ of young adults may become daily smokers resulting in increased smoking intensity and cumulative cigarette consumption (Yeh, Wang, Lin, \& Chung, 2014). Fortunately, according to Global Youth Tobacco Survey of Taiwan, the smoking prevalence of senior high school students gradually reduced from $20 \%$ to $13 \%$ (since 2011 to 2014). This declining prevalence could be credited with early anti-smoking legislation including expanding smoke-free areas, displaying six possible graphic warning labels, banning tobacco advertising, and adding surcharge of each cigarette packet in Taiwan, which drastically raised the awareness of the harm of smoking and cessation (Chiang \& Chang, 2016). However, we still have room for improvement in smoking cessation when comparing with the United States (US). Deferring and delaying smoking onset as well as promoting early cessation in adolescence can curb the tobacco epidemic and provide more broadly health benefits through higher educational attainment (D’Angelo et al., 2016; Sabado et al., 2017).

According to the treatment guideline, nicotine dependent smokers need support to quit smoking with combination therapy (counseling and smoking cessation medicine) as the most effective (Poulsen et al., 2015). Nicotine replacement therapy (NRT, patch, gum, or other formulations) offsetting nicotine craving, bupropion blunting nicotine withdrawal symptoms and varenicline lessening both nicotine craving and withdrawal symptoms are common medications used individually or in combination to achieve better likelihood of abstinence at six months or more than no intervention and have been recommended by the US Preventive Services Task Force. However, unaffordable cost, potential side effects of the pharmacotherapy such as mouth, throat and nasal irritation, dry mouth, dyspepsia, cardiovascular distress and insomnia may lead to a premature discontinuation of the medications and low abstinence rates (Thanavaro \& Delicath, 2010; Chang, Fung, Li, Lin, Tam, Tang, \& Tong, 2013). NRT may have the potential for priming the brain for nicotine addiction since the adolescent are highly susceptible to nicotine even early or limited nicotine exposure and may also lead to other drug addiction (Karpinski, Timpe, \& Lubsch, 2010). Serious neuropsychiatric symptoms induced by varenicline, suicide thoughts and attempts, hostility or behavior change for example, were reported in some studies and must be taken into consideration while prescribing the drug to specific patients (Thanavaro \& Delicath, 2010). 
Besides routine intervention, acupuncture, auricular acupuncture (AA), and electrical stimulation are also used widely owing to their effectiveness and safety for smoking cessation. The use of AA in smoking cessation has been increasing during past 20 years, and is often given with behavior therapy by advice in health and social welfare facilities (Kang, Shin, Kim, \& Youn, 2005). Superior results of AA regarding abstinence rate, $\mathrm{CO}$, and conitine during follow-up period were showed in two studies (Yeh et al., 2014; Fritz, Carney, Steinmeyer, Ditson, Hill, \& Zee-Cheng, 2013). A meta-analysis concluded that auriculotherapy may be effective in smoking cessation attributable to psychological factors but not related to the location point (Thanavaro \& Delicath, 2010; Silva Rde, Chaves Ede, Pillon, Silva, Moreira Dda, \& Iunes, 2014). Additionally, a smoking cessation rate can increase up to nearly $30 \%$ in AA group with educational counseling comparing with AA alone, which implies cooperation of these strategies may impose synergistic effect, though still lack of more rigorous, well-designed and large sample size studies (Thanavaro \& Delicath, 2010). From the perspectives of safety and accessibility for, AA offers a simple, non-invasive, and long-term constancy method for smoking cessation (Yeh, Chang, Chu, \& Chen, 2009).

This observational study aims to assess the efficacy and safety of AA integrated with intense group counseling in assisting the senior high school students aged from 15 to 18 to quit smoking and relieve nicotine withdrawal symptoms.

\section{Methodology}

\section{Study Design and Population}

Data are from an observation study of 40 students aged 15-18 years old and studying in a senior high school in the local metropolitan area. The whole course will be executed for 11 weeks with 6-week intervention and 1 month follow-up period. Each student had smoked continuously before joining the course and had no health problems. They may be recruited by their own choice or mandatory attendance because of violating regulation about smoking-free campus. This study obtained informed-consent forms from all the participants. Data collected would be confidential and de-linked and the participants were free to withdraw from study at any time.

\section{Interventions}

Participants will receive AA and group counseling on a weekly basis during the 6-week treatment period. All treatments will be performed by the same registered acupuncturist who practiced for more than five years and are specialized in AA to ensure participant consistency and reliance of treatment. AA points including shenmen, lung, stomach, mouth, and endocrine, which are known to be effective in smoking cessation (Figure 1) or nonspecific ear acupoints are used for each participant according to their discomfort condition and acceptability (Yeh et al., 2014; Kang et al., 2005; Yeh et al., 2009). There will be no skin penetration by a seed-embedding pellet utilizing Wanbuliuxingzi seeds. Each pellet is around $1.2 \mathrm{~mm}$ in diameter and attached to a round adhesive tape ( $7 \mathrm{~mm}$ in diameter) in a tan colour which is close to skin color. The skin surface will be sterilized with a $75 \%$ alcoholic swab before five pellets are taped on the specific area. Once taped on the ear, the acupuncturist will press each pellet for $10 \mathrm{sec}$, or until the ear became reddish and/or slightly sore. The participants will be instructed to press the pellets three times (may be before or after meals) at least 60 seconds a day by themselves throughout the week and whenever they feel a craving for cigarettes, which may induce emotional distress, depressed mood anxiety, and sleep disturbance. Pellet(s) that drop off during the week will 
not be replaced and the remaining pellets will be removed by the acupuncturist at the subsequent visit. The two ears are used simultaneously in order to enhance and prolong efficacy.

The format of group counseling contained files of text, animation, graphics, picture, sound, image or advertising video about smoking to provide unhindered interaction between participants and the professions. The major contents of the counseling included the impact of smoking on health and the environment, information about smoking hazards, strategies for smoking cessation, and problems arising during the smoking cessation period and their solutions. Moreover, the professions will develop an individualized approach to smoking cessation based on nicotine dependency scores, unique triggers, and types of smokers. Participants were informed of what types of smokers they were classified as and this information was also used to develop individual strategies for smoking cessation. The participants also can share and discuss former experience and insights regarding smoking cessation, no matter successful, fail, right, or wrong, which may inspire the peers to take actions. The counseling session usually required 45-60 minutes of face-to-face discussion with each participant and be held after the assessment and AA intervention.

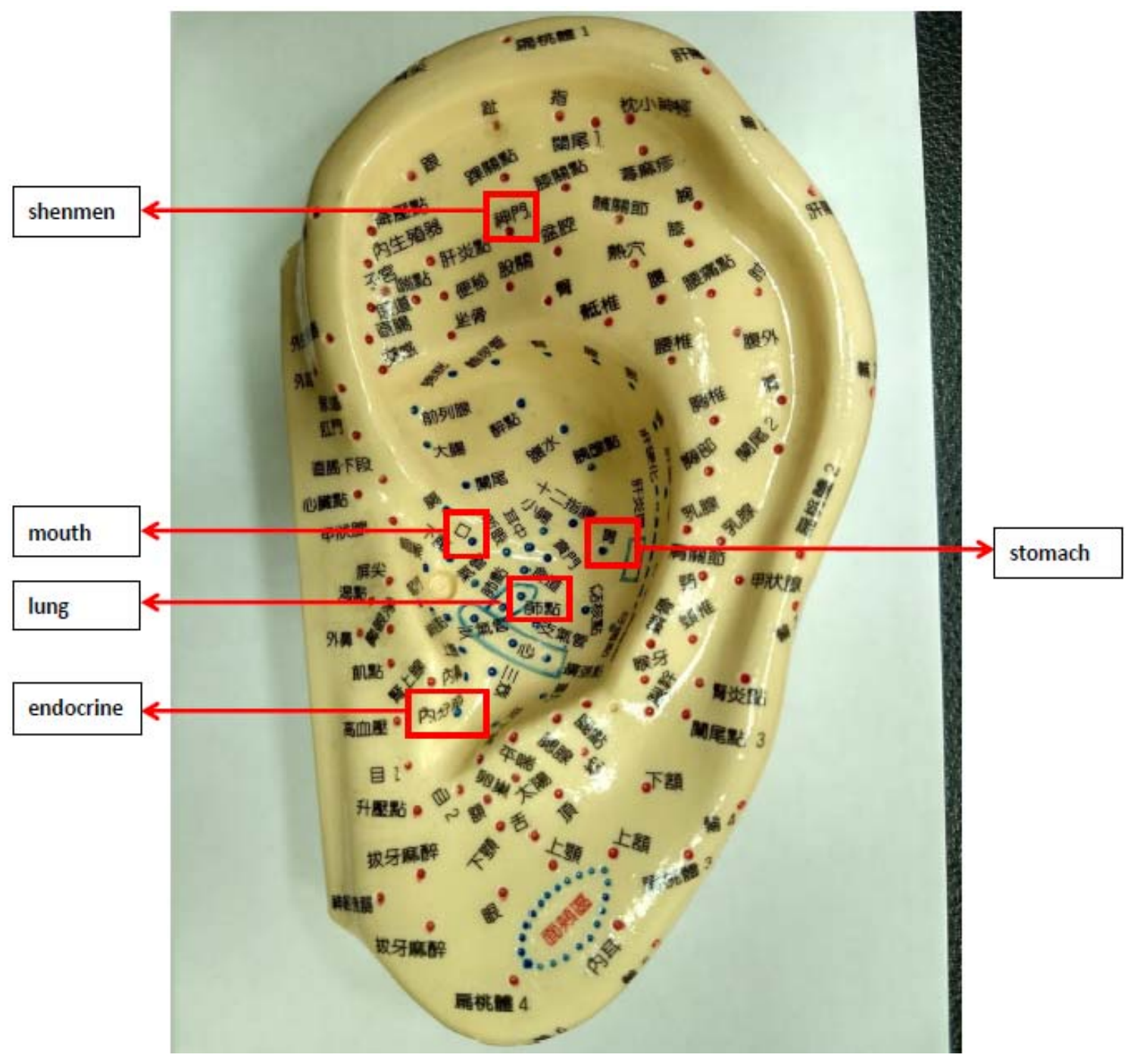

Figure 1. Auricular acupoints known to be effective in smoking cessation. 


\section{Measurement}

General characteristics. We collected information on gender, age, age of initial smoking, age of regular smoking, and number of cigarettes smoked per day

Smoking status. Current smokers who smoked every day or some days were the target participants.

Fagerstrom test for nicotine dependence. Severity of nicotine dependence was measured by the Fagerstrom Test for Nicotine Dependence (FTND) every week. We used the standard questions and instructed by well-trained staffs. Each answer will correspond to a point and the sum these points can indicate the dependent severity of the participants. The higher likelihood of nicotine dependence was indicated by higher score.

- How soon after you wake do you smoke your first cigarette?

- Do you find it difficult to refrain from smoking in places where it is forbidden (i.e., church, school, work, etc.)?

- Which cigarette would you hate most to give up?

- How many cigarettes per day do you typically smoke?

- Do you smoke more frequently during the first hours after waking up than during the rest of the day?

- Do you smoke even if you are so ill that you are in bed most of the day?

Carbon monoxide exhalation. A Micro Smokerlyzer carbon monoxide (CO) breath monitor (Type: EC50-MP, Bedfont Scientific Corporation, England) was used to determine CO exhalation as an objective variable recorded during each visit. The non-smoker should have concentration of $\mathrm{CO}$ exhalation less than 10 parts per million (PPM).

Abstinence rate. Abstinence was defined as no cigarette consumption at Week 6 and continuing to the next month. Meanwhile, the $\mathrm{CO}$ exhalation should exhibit within normal range as non-smokers at the last assessment. Abstinence rate represents the ratio of the success rate of smoking cessation among the participants.

\section{Statistical Analysis}

The data were analyzed by an SPSS version 18.0. Baseline characteristics of participants (age, gender, number of cigarettes smoked per day, history of packed-years) will be expressed as mean values \pm standard deviation (SD). The null hypothesis will be adopted in comparing the outcome measurement (FTND and CO exhalation). Repeated measures ANOVA test and P-value linear trend analysis were used to analyze the effects of AA in combination with group counseling which are related to changes in the taste of tobacco, desire to smoke, and reduction in cigarette consumption. There were initial 40 participants enrollment, however, not all of them complete the full course. In order to focus the efficacy of AA in combination with group counseling among small sample size and prevent bias from missing data, we only analyze the data from 23 students who entirely participated.

\section{Results}

\section{General Characteristics and Smoking Status}

The subjects in the present study were all male, and age. The age, age of initial smoking, and number of cigarettes smoked per day are listed on Table 1 . All the subjects were daily smokers. 
Table 1

General Characteristics $(N=23)$

\begin{tabular}{ll}
\hline Age constitution & Number of subjects \\
\hline 15 & 1 \\
16 & 1 \\
17 & 1 \\
18 & 19 \\
\hline Age of initial smoking & Age of subjects \\
\hline 9 & 2 \\
10 & 2 \\
11 & 3 \\
12 & 7 \\
13 & 8 \\
14 & 1 \\
\hline Number of cigarettes smoked per day & Number of subjects \\
\hline 0 & 0 \\
$1-5$ & 5 \\
$6-10$ & 6 \\
$>10$ & 12 \\
\hline
\end{tabular}

Comparison Between Fagerstrom Test for Nicotine Dependence, CO Exhalation and Consumption of Cigarettes

The difference among these outcome measurements are listed on Table 2 and Table 3 and all showed significant reduction $(\mathrm{P}<0.05)$ as time and interventions went on. However, the mean $\mathrm{CO}$ exhalation illustrated the most reduction at Week 5 and rebounded in Week 6. Additionally, the mean consumption of cigarettes rebounded at Week 5 and then returned to sustained reduction in the consecutive assessments (Figure 2, Figure 3, and Figure 4). In view of the declining amounts of these variables among Week 1, Week 6, and last assessment by pairwise comparison test, there are diverse results. First, all amounts of Week 6 and last assessment are significantly lower than Week $1(\mathrm{P}<0.05)$. Second, only FTND of last assessment showed a slight increase than Week 6 but without significance $(\mathrm{P}=0.503)$. Last, $\mathrm{CO}$ exhalation and consumption of cigarettes of last assessment showed significant reduction than Week 6, suspecting there were residual influence on smoking cessation even though no intervention was applied to.

Table 2

Effects of Auricular Acupuncture on FTND, CO Exhalation, and Self-Reported Cigarette Consumption Every Assessment

\begin{tabular}{llll}
\hline Outcome measure & FTND & CO exhalation & Daily cigarette consumption \\
\hline Sequence & & \\
1st & $4.913 \pm 0.412$ & $16.957 \pm 1.845$ & $10.043 \pm 0.965$ \\
2nd & $3.609 \pm 0.443$ & $14.304 \pm 1.796$ & $8.174 \pm 1.046$ \\
3rd & $3.304 \pm 0.379$ & $12.391 \pm 1.664$ & $7.652 \pm 1.021$ \\
4 th & $3.000 \pm 0.356$ & $11.913 \pm 1.764$ & $7.391 \pm 1.101$ \\
5 th & $2.783 \pm 0.377$ & $8.478 \pm 1.103$ & $8.348 \pm 1.036$ \\
6th & $2.000 \pm 0.281$ & $11.696 \pm 1.441$ & $7.261 \pm 0.996$ \\
7 th & $2.130 \pm 0.316$ & $8.696 \pm 1.267$ & $6.348 \pm 0.904$ \\
\hline
\end{tabular}

Notes. FTND: Fagerstrom test for nicotine dependence; CO: Carbon monoxide. 
Table 3

Variation and Trend of Outcome Within Subjects

\begin{tabular}{lll}
\hline Outcome measurement & & \\
\hline FNTD of 7 Assessments & $\mathrm{F}$ & $\mathrm{P}$ \\
Greenhouse-Geisser & 36.699 & $\mathrm{P}<0.001$ \\
Linear Trend & 98.852 & $\mathrm{P}<0.001$ \\
CO of 7 Assessments & & \\
Greenhouse-Geisser & 7.477 & $\mathrm{P}=0.001$ \\
Linear Trend & 20.277 & $\mathrm{P}<0.001$ \\
Cigarette Consumption of 7 Assessments & & \\
Greenhouse-Geisser & 4.072 & $\mathrm{P}=0.006$ \\
Linear Trend & 11.036 & $\mathrm{P}=0.003$ \\
\hline
\end{tabular}

Notes. FTND: Fagerstrom test for nicotine dependence; CO: Carbon monoxide.

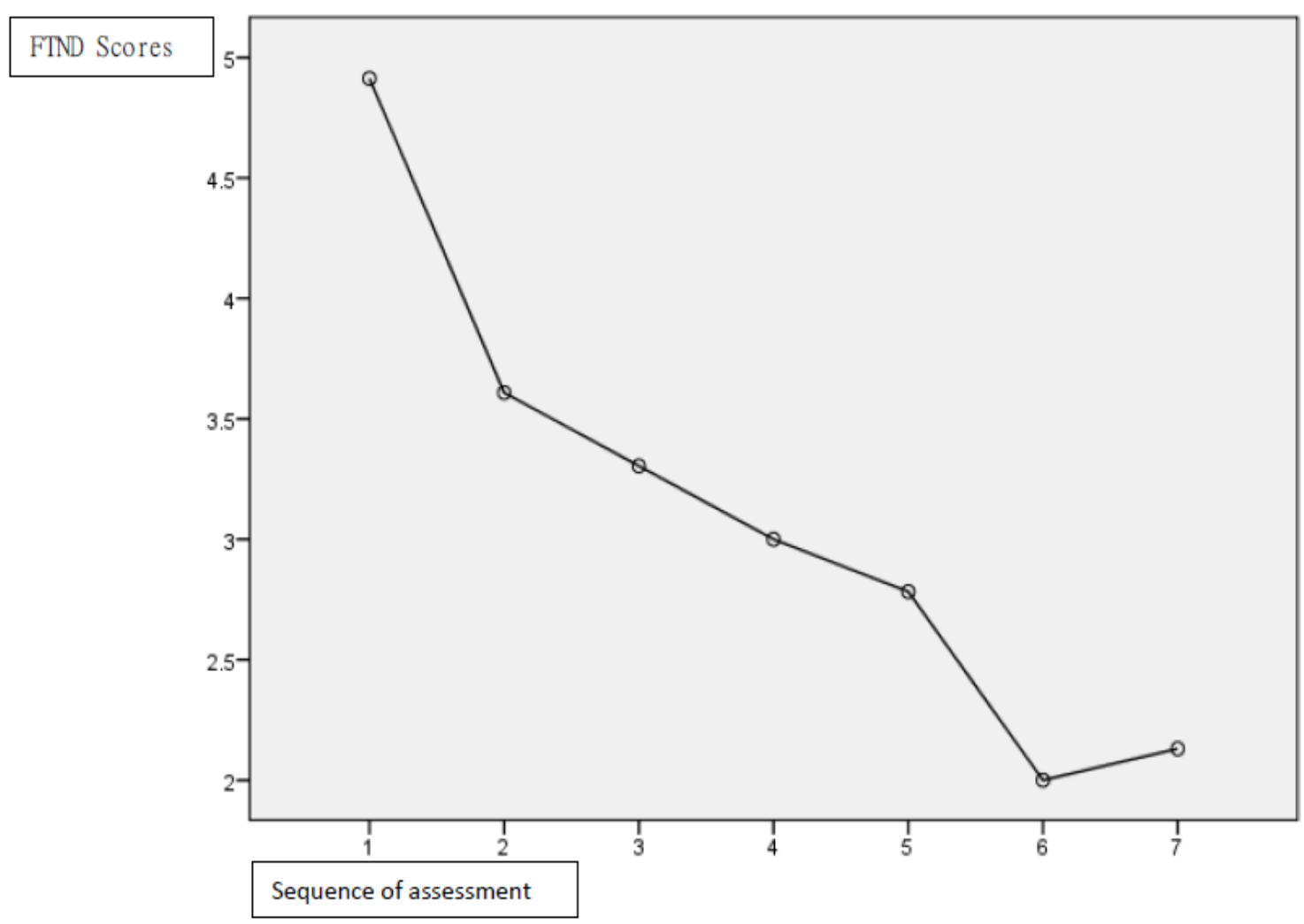

Figure 2. Pairwise comparison test of FTND scores. 


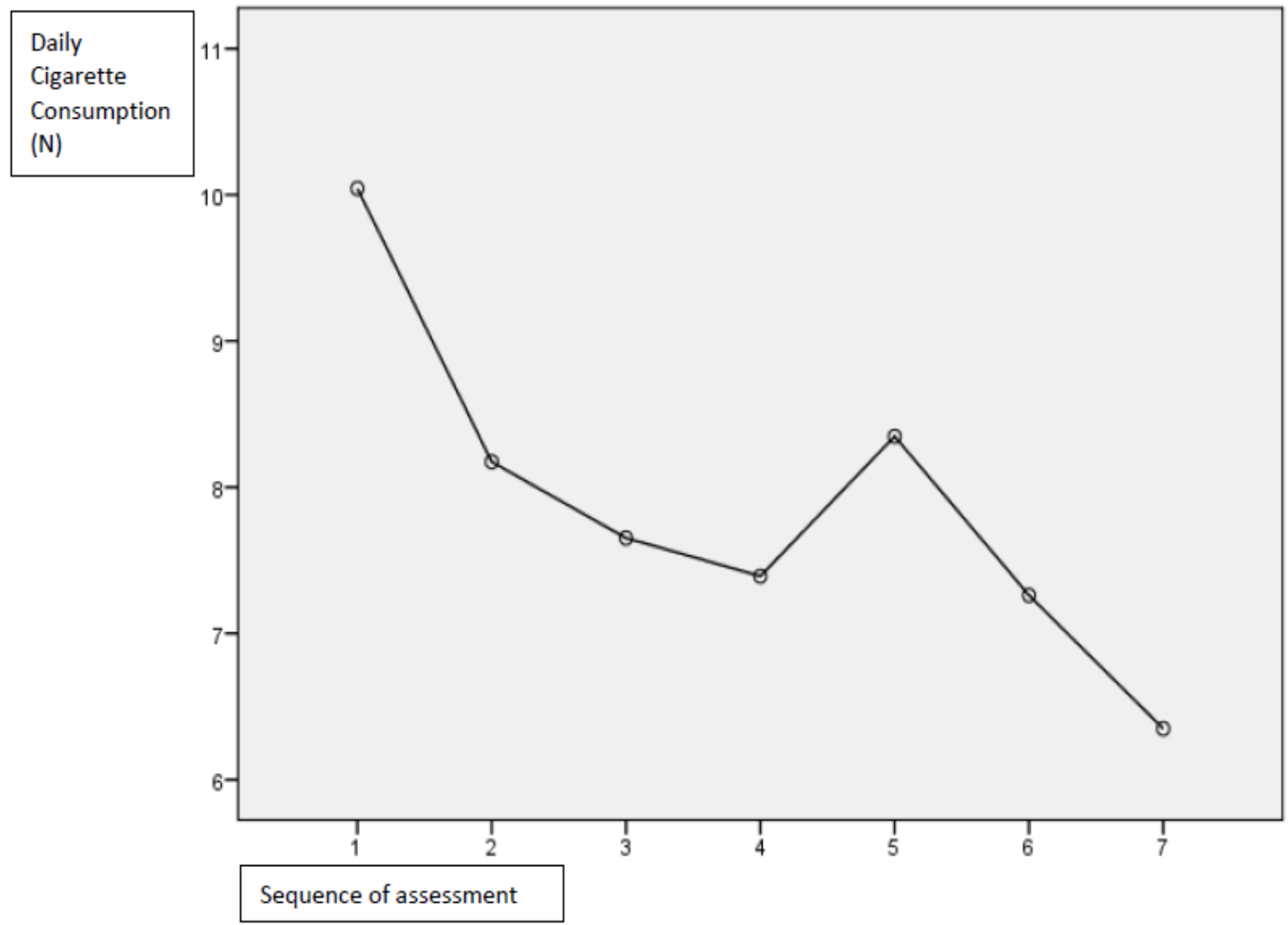

Figure 3. Pairwise comparison test of daily cigarette consumption.

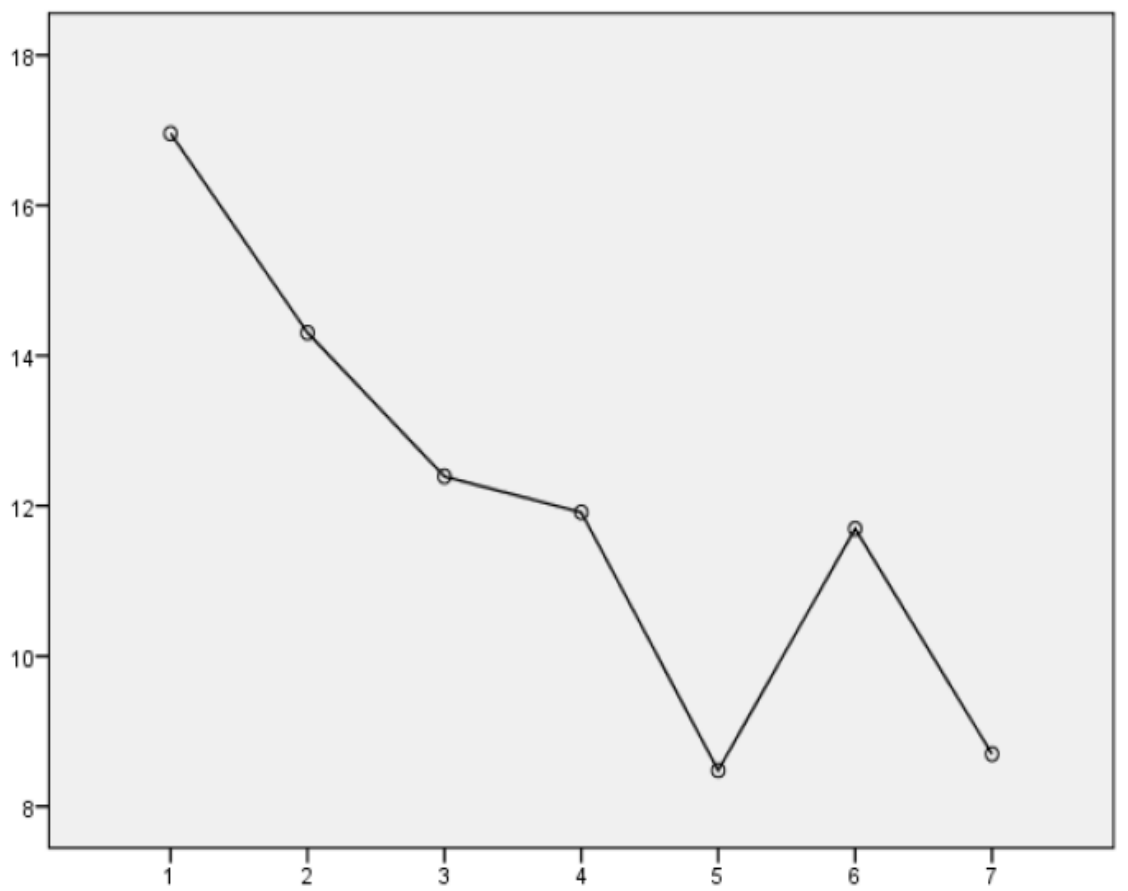

Sequence of assessment

Figure 4. Pairwise comparison test of $\mathrm{CO}$ exhalation. 


\begin{abstract}
Abstinence Rate
Among 23 subjects, only three of them achieved the goal of abstinence. Therefore, the abstinence rate can be calculated as $13 \%$.
\end{abstract}

\title{
Discussion
}

Most participants had their first smoking experience at an age before 15, which makes adolescents a special target for smoking prevention projects (Ekpu \& Brown, 2015). The earlier one starts to smoke, the more likely one's tobacco usage increases, making smoking cessation even harder (Yeh et al., 2009). This study found the positive effect of AA on smoking cessation for high school students especially in FTND, CO exhalation, and cigarette consumption, which presented consistent results with other studies (Yeh et al., 2014; Tucker, Ellickson, Orlando, \& Klein, 2005). Since AA lowered cigarette consumption, the technique can certainly bring benefits to adolescent health and future status, which had been proved by a prospective study stating that reducing the number of cigarettes smoked per day may incline by $15 \%$ the risk of mortality and $23 \%$ the mortality from cardiovascular problems (Silva Rde et al., 2014).

We used AA, rather than conventional body or extremity acupuncture for the purpose of safety (non-invasive and few adverse effects), intention to have a durable 1-week acupuncture effect and increase in subjects' compliance (Silva Rde et al., 2014; Wu, Chen, Liu, Lin, \& Hwang, 2007). AA is a simple and noninvasive intervention which can increase endorphin, and serotonin transmitted to the brain and corresponding organs in the rest of the body through nerves and meridian so as to modulate physiological reactions (Yeh et al., 2014). The elevating endorphin and rebalance of brain neurotransmitters can potentially eradicate symptoms nicotine reliance which are as effective as nicotine replacement therapy and help smokers remind of their terrible first experience of cigarette (Yeh et al., 2009). Another suspect mechanism could be placebo effect or ritualized function producing biological effect of a nonspecific noxious central inhibitory effect not relevant to the selected acupoints (Wu et al., 2007). These theories support our results that AA in combination with group counseling can significantly lower FTND and cigarette consumption through AA-induced release of endogenous opioid peptide.

Schools have been identified as an ideal site to deliver tobacco prevention programs since they capture the majority of youth across a large age range, especially the ages when most young people initiate smoking. High cost effectiveness is another advantage as the government could expect to save US\$13,316 per life year saved and a saving of US8,482 per quality-adjusted life years saved. The main perceived advantages of school-based group counseling are that almost all adolescents can be reached through schools, and a focus on education fits legitimately with the daily activities of schools and helps smokers accelerate the process of behavior change, improving positive cognition and attitude toward smoking cessation (Yeh et al., 2014; Ekpu \& Brown, 2015). Although numerous school-based smoking prevention and cessation studies have found short-term decreases in smoking prevalence by up to $30 \%-70 \%$, there still lack evidence on the long-term effectiveness of such programs. While longer and intense counseling sessions can assess smokers' readiness to quit, explore smoking habits and identify barriers to smoking cessation as well as factors leading to relapse, which assist professions to establish individualized quitting strategies for different smoker (Thanavaro \& Delicath, 2010). In comparison with single intervention, the addition of school-based group counseling may be needed to reinforce the success rate in abstinence and cigarette consumption of subjects who undergo AA for smoking cessation. 
However, there are some limitations of the study. First, the study used small sample size $(\mathrm{N}=23)$ and the subjects were limited in a restricted area, such as school, which may cause low statistical power and selection bias. Second, CO exhalation was used as an objective outcome measurement, however, its poor sensitivity of $\mathrm{CO}$ as indicators of smoking activity has been reported in young smokers. Other confirmative indicators are required because $\mathrm{CO}$ exhalation is influenced easily by different physical activity, rate of pulmonary ventilation, and dwelling environment (Yeh et al., 2014). Third, from all of these studies regarding smoking cessation, an issue that remains unresolved is the extent to which reductions in adolescent smoking lead to lower smoking prevalence and/or earlier smoking cessation in adulthood. Our study only had three persons achieving abstinence with normal $\mathrm{CO}$ exhalation and self-report zero cigarette consumption. Nonetheless, this was only a 6-week course plus 1 month follow-up period, the relapse rate in the future, authentic rate, and duration of cigarette abstinence and cannot be determined, given that generally 6-month follow-up is needed in most well-designed studies (Thanavaro \& Delicath, 2010; Kang et al., 2005; Ekpu \& Brown, 2015). Last, the present study is not a randomized controlled, double-blinded, and plcebo-controlled clinical trial, which may lead to performance, attrition, and detection bias. In light of this, our results should be interpreted cautiously and future researches must prevent selection bias, treatment assignment bias resulting in Hawthrone effect and be designed as randomized controlled trial to validate the impact of AA on smoking cessation.

\section{Conclusion}

Full implementation of comprehensive tobacco prevention and sustained control interventions that restrict youths' access to tobacco products and reduce exposure to youth-oriented tobacco product promotions can reduce tobacco use among adolescents (D'Angelo et al., 2016; Jamal et al., 2017). Nicotine replacement therapy such as patch or gum bupropion as well as counseling are advised in smoking cessation even though the success rate are still unsatisfactory (Karpinski, Timpe, \& Lubsch, 2010; Wu et al., 2007). In Taiwan, despite varenicline user reported higher abstinence rate than NRT patch users, the serious neuropsychiatric symptoms observed in adult patients may limit scope of application (Chang, Lo, Chang, Hsueh, \& Tsai, 2016). The study showed AA in combination with group counseling is a safe intervention which can lower nicotine reliance and daily tobacco consumption for smoking cessation. We suggest AA can be regarded as an adjuvant therapy associated with other types of intervention or as a major alternative to enhance the benefits in those who are trying to quit smoking (Thanavaro \& Delicath, 2010; Silva Rde et al., 2014).

\section{References}

Andrade, R. C. C., Ferreira, A. D., Ramos, D., Ramos, E. M. C., Scarabottolo, C. C., Saraiva, B. T. C., Gobbo, L. A., \& Christofaro, D. G. D. (Nov.-Dec. 2017). Smoking among adolescents is associated with their own characteristics and with parental smoking: Cross-sectional study. Sao Paulo Med J, 135, 561-567.

Chang, E., Fung, L. C., Li, C. S., Lin, T. C., Tam, L., Tang, C., \& Tong, E. K. (Sep. 2013). Offering acupuncture as an adjunct for tobacco cessation: A community clinic experience. Health Promot Pract, 14, 80s-87s.

Chang, P. Y., Lo, P. C., Chang, H. C., Hsueh, K. C., \& Tsai, Y. W. (2016). Comparative effectiveness of smoking cessation medications: A national prospective cohort from Taiwan. PLoS One, 11, e0166992.

Charlton, A. (2004). Medicinal uses of tobacco in history. Journal of the Royal Society of Medicine, 97, 292-296.

Chiang, C. Y., \& Chang, H. Y. (2016). A population study on the time trend of cigarette smoking, cessation, and exposure to secondhand smoking from 2001 to 2013 in Taiwan. Popul Health Metr, 14, 38.

D’Angelo, D., Ahluwalia, I. B., Pun, E., Yin, S., Palipudi, K., \& Mbulo, L. (Sep. 2, 2016). Current cigarette smoking, access, and purchases from retail outlets among students aged 13-15 years-Global youth tobacco survey, 45 countries, 2013 and 2014. MMWR Morb Mortal Wkly Rep, 65, 898-901. 
Ekpu, V. U., \& Brown, A. K. (2015). The economic impact of smoking and of reducing smoking prevalence: Review of evidence. Tob Use Insights, 8, 1-35.

Fritz, D. J., Carney, R. M., Steinmeyer, B., Ditson, G., Hill, N., \& Zee-Cheng, J. (Jan.-Feb. 2013). The efficacy of auriculotherapy for smoking cessation: A randomized, placebo-controlled trial. J Am Board Fam Med, 26, 61-70.

Haug, S., Paz Castro, R., Kowatsch, T., Filler, A., \& Schaub, M. P. (Nov. 2017). Efficacy of a technology-based, integrated smoking cessation and alcohol intervention for smoking cessation in adolescents: Results of a cluster-randomised controlled trial. J Subst Abuse Treat, 82, 55-66.

Jamal, A., Gentzke, A., Hu, S. S., Cullen, K. A., Apelberg, B. J., Homa, D. M., \& King, B. A. (Jun. 16, 2017). Tobacco use among middle and high school students-United States, 2011-2016. MMWR Morb Mortal Wkly Rep, 66, 597-603.

Kang, H.-C., Shin, K.-K., Kim, K.-K., \& Youn, B.-B. (2005). The effects of the acupuncture treatment for smoking cessation in high school student smokers. Yonsei Medical Journal, 46, 206-212.

Karpinski, J. P., Timpe, E. M., \& Lubsch, L. (Oct.-Dec. 2010). Smoking cessation treatment for adolescents. The Journal of Pediatric Pharmacology and Therapeutics: JPPT, 15, 249-263.

Pedro, J. M., Brito, M., \& Barros, H. (2017). Tobacco consumption and nicotine dependence in Bengo Province, Angola: A community-based survey. PLoS One, 12, e0188586.

Poulsen, P. B., Spillemose, H., Nielsen, G., Hergel, L. L., Wedell-Wedellsborg, D., Strand, M., \& Ringbak, T. (Feb. 2015). Real-life effectiveness of smoking-cessation treatments in general practice clinics in Denmark. The escape smoke project. Respir Med, 109, 218-227.

Sabado, M. D., Haynie, D., Gilman, S. E., Simons-Morton, B., \& Choi, K. (Dec. 2017). High school cigarette smoking and post-secondary education enrollment: Longitudinal findings from the NEXT Generation Health Study. Prev Med, 105, 250-256.

Silva Rde, P., Chaves Ede, C., Pillon, S. C., Silva, A. M., Moreira Dda, S., \& Iunes, D. H. (Oct. 2014). Contributions of auriculotherapy in smoking cessation: A pilot study. Rev EsC Enferm USP, 48, 883-890.

Thanavaro, J. L., \& Delicath, T. A. (2010). Auricular transcutaneous electrostimulation therapy and intensive counseling for the treatment of smoking cessation in a primary care practice. Journal of Addictions Nursing, 21, 215-224.

Tucker, J. S., Ellickson, P. L., Orlando, M., \& Klein, D. J. (Aug. 2005). Predictors of attempted quitting and cessation among young adult smokers. Prev Med, 41, 554-561.

Vickerman, K. A., Zhang, L., Malarcher, A., Mowery, P., \& Nash, C. (Jul. 16, 2015). Cessation outcomes among quitline callers in three states during a national tobacco education campaign. Prev Chronic Dis, 12, E110.

Wu, T. P., Chen, F. P., Liu, J. Y., Lin, M. H., \& Hwang, S. J. (Aug. 2007). A randomized controlled clinical trial of auricular acupuncture in smoking cessation. J Chin Med Assoc, 70, 331-338.

Xiao, L., Feng, G. Z., Jiang, Y., Zhang, J. R., \& Liu, L. X. (May 10, 2017). Tobacco use rate and associated factors in middle school students in China. Zhonghua Liu Xing Bing Xue Za Zhi, 38, 567-571.

Yeh, M. L., Chang, C. Y., Chu, N. F., \& Chen, H. H. (2009). A six-week acupoint stimulation intervention for quitting smoking. Am J Chin Med, 37, 829-836.

Yeh, M. L., Wang, P. L., Lin, J. G., \& Chung, M. L. (2014). The effects and measures of auricular acupressure and interactive multimedia for smoking cessation in college students. Evid Based Complement Alternat Med, 2014, 898431. 